\title{
Conceptual design of the beryllium rotating target for the ESS-Bilbao facility
}

\author{
S. Terrón ，F. Sordo ，M. Magán , A. Ghiglino ，F. Martínez ，P.J. de Vicente \\ R. Vivanco ，K. Thomsen , J.M. Perlado , F.J. Bermejo , A. Abánades
}

\begin{abstract}
A B S T R A C T
The ESS-Bilbao facility, hosted by the University of the Basque Country (UPV/EHU), envisages the operation of a high-current proton accelerator delivering beams with energies up to $50 \mathrm{MeV}$. The timeaveraged proton current will be $2.25 \mathrm{~mA}$, delivered by $1.5 \mathrm{~ms}$ proton pulses with a repetition rate of $20 \mathrm{~Hz}$. This beam will feed a neutron source based upon the Be $(p, n)$ reaction, which will enable the provision of relevant neutron experimentation capabilities. The neutron source baseline concept consists in a rotating beryllium target cooled by water. The target structure will comprise a rotatable disk made of 6061-T6 aluminium alloy holding 20 beryllium plates. Heat dissipation from the target relies upon a distribution of coolant-flow channels. The practical implementation of such a concept is here described with emphasis put on the beryllium plates thermo-mechanical optimization, the chosen coolant distribution system as well as the mechanical behavior of the assembly.
\end{abstract}

\section{Introduction}

The advent of important developments in accelerator technology as well as the dissemination of neutronic design capabilities has enabled the construction of small to medium scale acceleratordriven neutron facilities which, are nowadays playing a significant role in the development and optimization of large facilities. In fact, a number of small-to-medium range installations worldwide are now in operation, under construction or planned. The role of these facilities is glaringly shown by work performed on moderator and instrument development carried out at laboratories such as the Low Energy Neutron Source (LENS) facility at the University of Indiana (EE.UU) in relation to the SNS project (Oak Ridge National Laboratory, EE.UU) $[1,2]$, or the activities developed within the Hokkaido University electron linac regarding the MLF J-PARC facility (Tokai, Japan) [3]. In addition, a number of neutron facilities are now under construction mostly relying on proton accelerators as drivers. Most of them cover a wide spectrum of activities which, apart from neutron scattering purposes, also envisage activities in other areas such as Accelerator Driven Systems (ADS) for waste transmutation, hadron therapy, neutron imaging, etc.
The developments just referred to open up new opportunities for university scale organizations to enter the field of neutron physics with modest investments, carrying out activities within fields as diverse as materials science, nuclear physics, medical physics, engineering and cultural heritage [4]. As a matter of fact, small to medium power sources may provide invaluable experimental resources, useful for the development of neutron techniques and training of the neutron users and accelerator and source operators. Not all measurements or experiments require the beam intensity offered by the high power sources, and excellent science programs can be carried out at smaller facilities. Small sources can be adapted to a specialized community, which may better reflect the regional requirements. Scientific and technological experiences and know-how developed at such sources are shared effectively with the larger facilities. Hence, covering all the needs of the user community, low, medium and high power neutron sources should be considered as complementary, each playing an important role in the application and advancement of neutron techniques, as recently emphasized by a report of a working group of the Institut Laue Langevin (ILL) associates [5].

Within the above sketched framework, the ESS-Bilbao project seeks to channel the Spanish contribution to the European Spallation Source (ESS) project [6], as well as to develop in-house capabilities in accelerator science and technology. For such an avail, a compact although high power proton and ${ }^{-} \mathrm{H}$ accelerator is 
Table 1

ESS-Bilbao accelerator main parameters.

\begin{tabular}{ll}
\hline Proton energy & $50 \mathrm{MeV}$ \\
Peak current & $75 \mathrm{~mA}$ \\
Repetition rate & $20 \mathrm{~Hz}$ \\
Pulse length-variable & $1.5 \mathrm{~ms}$ \\
Average current & $2.25 \mathrm{~mA}$ \\
Average power & $112 \mathrm{~kW}$ \\
\hline
\end{tabular}

Table 2

Neutron Yield for different target materials for $50 \mathrm{MeV}$ protons, calculated using ENDEF-VII/B [10] cross-sections.

\begin{tabular}{llc}
\hline Material & N/p & Av. energy (MeV) \\
\hline Carbon & $7.5 \times 10^{-3}$ & 8.0 \\
Lithium & $4.3 \times 10^{-2}$ & 13.3 \\
Beryllium & $6.5 \times 10^{-2}$ & 7.8 \\
\hline
\end{tabular}

now under construction. The machine has been designed to feed several irradiation laboratories as well as a neutron generation target, the design of which constitutes the issue of the present work.

\section{Conceptual target design}

The accelerator under construction in Bilbao will drive a compact neutron source whose design will be based on a rotating beryllium target cooled by water. The neutron yield expected for such a medium-sized source comes to about $\sim 10^{15} \mathrm{n} / \mathrm{s}$ [7]. Such a neutron fluence is however large enough to carry out relevant experimental tasks such as testing components and subsystems (moderators, neutron guides, neutron detectors, choppers or even instrument concepts) to be installed at the ESS facility, develop projects in collaboration with other large-scale facilities, as well as to train local neutron scattering users. The main parameters of the ESS-Bilbao accelerator are summarized in Table 1.

A glance to data about the accelerator design parameters of several neutron sources compiled in Ref. [7], shows that the beam delivered by the ESS-Bilbao machine largely differs from those employed in neutron production facilities based upon spallation reactions, and on the other hand, delivers significantly more power than that handled at other university-scale neutron facilities such as LENS. Nevertheless, it falls within the range of those used to build low energy converters for the generation of radioactive ion beams like SPIRAL2 [8] at GANIL (Caen, France) or INFN [9] (Legnaro, Italy). For these low energy targets, lithium, beryllium or carbon (graphite) are the materials of choice. The latter is preferred due to its reasonable production efficiency for stripping $(d, n)$ reactions, its very good thermo-mechanical properties and the possible use of heat radiation cooling for moderate ion currents. However for $50 \mathrm{MeV}$ protons the neutron yield of a carbon target is about one order of magnitude lower than that provided by other light elements such as lithium or beryllium (Table 2). Besides, since the main purpose of the ESS-Bilbao target is to deliver thermal and cold neutron beams, thermal or cryogenic moderators must be placed as close as possible to the target [7], precluding the use of radiation cooling and making less interesting the use of carbon. This, together with the better mechanical properties of beryllium and its easier handling compared with metallic lithium and other lithium-based alloys, makes beryllium the material of choice.

As regards the cooling of the ESS-Bilbao target, a low-pressure flow of water appears as the most adequate option. Such a coolant ensures the necessary heat-removal capacity given the thermal power densities involved [11], and presents several other advantages like the existing operational experience within nuclear industry, the availability of off-the-shelf nuclear and vacuumgrade circuit components (pumps, feedthroughs, seals, etc.), and the possibility of hands-on maintenance of parts of the cooling loop. As an added bonus, the design here considered may profit from the experience of other beryllium-water based facilities, such as in operation at LENS [12] or the system built at the National Institute of Radiological Sciences (NIRS) Center (Chiba, Japan) [13]. However both are stationary targets which operate at a far lower power ( $8 \mathrm{~kW}$ for LENS and $3.2 \mathrm{~kW}$ for NIRS) than that delivered by the ESS-Bilbao accelerator. Assuming that the maximum heat flux which can be removed by single-phase (non-boiling), low pressure water is about $1 \mathrm{MW} / \mathrm{m}^{2}\left(h \approx 10^{4} \mathrm{~W} / \mathrm{m}^{2} \mathrm{~K}, T_{\text {surf }}-T_{\text {bulk }} \approx 100 \mathrm{~K}\right)$ and given the ESS-Bilbao beam power density $\left(1.12 \times 10^{5} \mathrm{~W}\right.$ and $<10^{-2} \mathrm{~m}^{2}$ cross-section), the implementation of a rotating target design becomes mandatory; even if the sharply pulsed nature of the heat load is neither explicitly taken into account nor the associated thermo-mechanical effects considered.

\section{ESS-Bilbao target description}

On the grounds of considerations given above, the design of the ESS-Bilbao target will consist of a rotating disk holding watercooled beryllium plates as it is sketched in Fig. 1. The conceptual design of such a target benefits from the analysis done during the design phase of other rotating target projects such as the second target station project SNS [14] or that regarding the German SNQ project [15], and from the experience acquired during the manufacturing of the rotating target flow test stand [16]. This test stand, built and operated within the ESS-Bilbao facilities, simulates the solid rotating design option for the second target station of SNS, within the framework of a collaborative agreement established between the two institutions.

The structural material of the disk will be the 6061-T6 aluminium alloy. The inner cooling circuit will be basically defined by the space between the disk components. The coolant enters into the disk through its axis, is then distributed radially to the beryllium plates through the upper distribution channels, gets back towards the disk center through the collection of lower channels, and leave it coaxially through the disk axis as shown in Fig. 2. The whole disk assembly is about $2 \mathrm{~m}$ in diameter and $15 \mathrm{~cm}$ thick.

To ensure that every beam pulse hits a different beryllium plate, the accelerator and target will be synchronized using for the purpose information provided by the installation master clock and

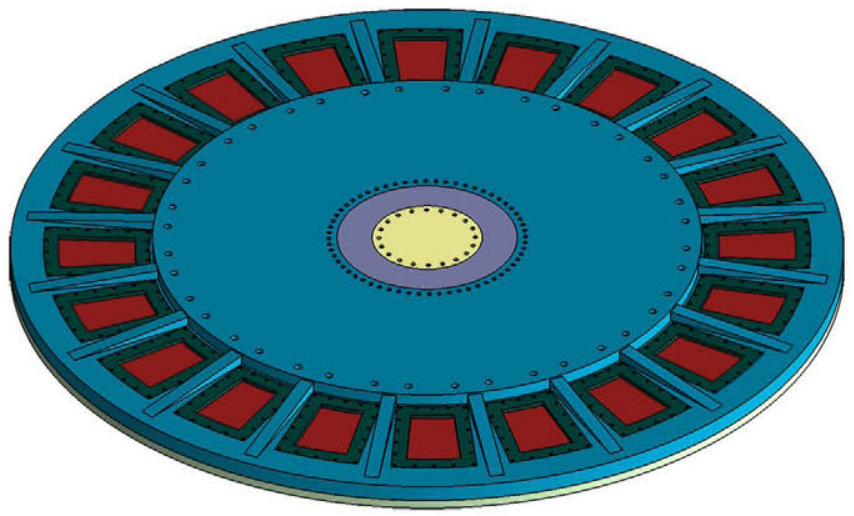

Fig. 1. ESS-Bilbao target overview. 
(a)

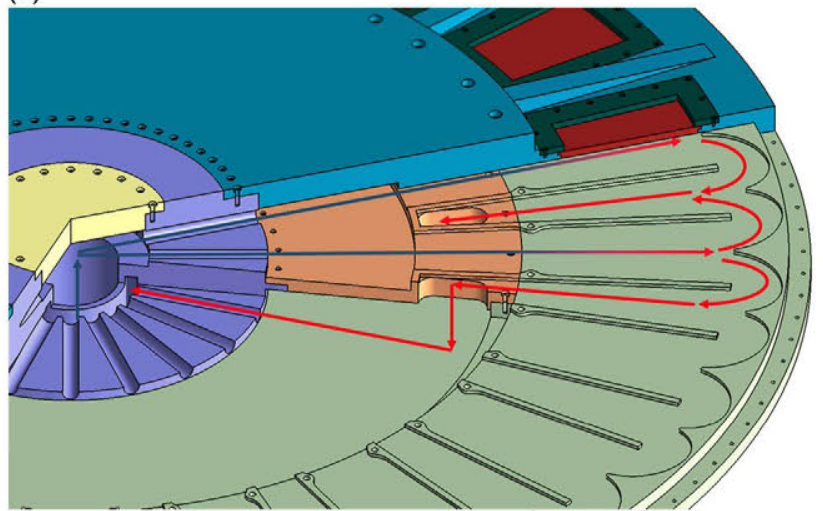

(b)

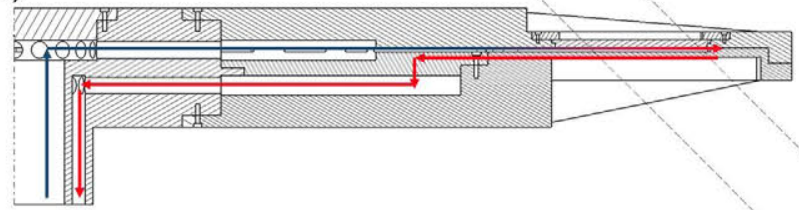

Fig. 2. ESS-Bilbao water flow scheme.

readings of the disk velocity and phase taken from sensors. The necessary control loops and interlock system will be set up.

As regards, the moderator-reflector assembly, the neutronic analysis performed so far and reported in Ref. [7], shows that the best option for this target concept relies on a configuration consisting of a methane moderator in slab configuration, surrounded by a beryllium reflector. The angle between the proton beam and the beryllium plates surface is $45^{\circ}$. This angle is chosen to provide a beam footprint of elliptical shape thus better distributing the incoming protons over the plate surface [7]. The whole assembly is covered by several layers of shielding materials in order to reduce the dose rate, an arrangement that is depicted in Fig. 3. The current assembly has been designed to hold up to two moderators with several cold and thermal neutron lines each, plus a fast neutron line in the proton incidence direction. This facility will be optimized for cold neutrons production and will constitute a flexible facility able to test ESS components with a cold neutron flux scaled down by a factor of 150800 regarding the one expected in ESS [7].

The next sections summarize the design process supporting this target concept, which profits from advantages of rotating targets as well as with the relatively moderate engineering requirements of low energy neutron sources.

\section{Design considerations}

\subsection{Beryllium elements}

The specification of the detailed dimensions of the beryllium plates requires to define the element thickness, to decide how many of them the target will hold and to estimate how long their lifespan will be.

As far as the definition of the beryllium plate thickness, three main strongly coupled factors must be taken into account such as the neutronic performance, the thermo-mechanical effects and radiation damage due to gas bubbles resulting from nuclear reactions and subsequent implantation within the material.

The incoming protons loose energy along their ionization tracks within the beryllium plates and show a strong Bragg peak just before they come to rest. For $50 \mathrm{MeV}$ protons such a peak appears after a track of about $15 \mathrm{~mm}$ within the solid. Taking into (a)

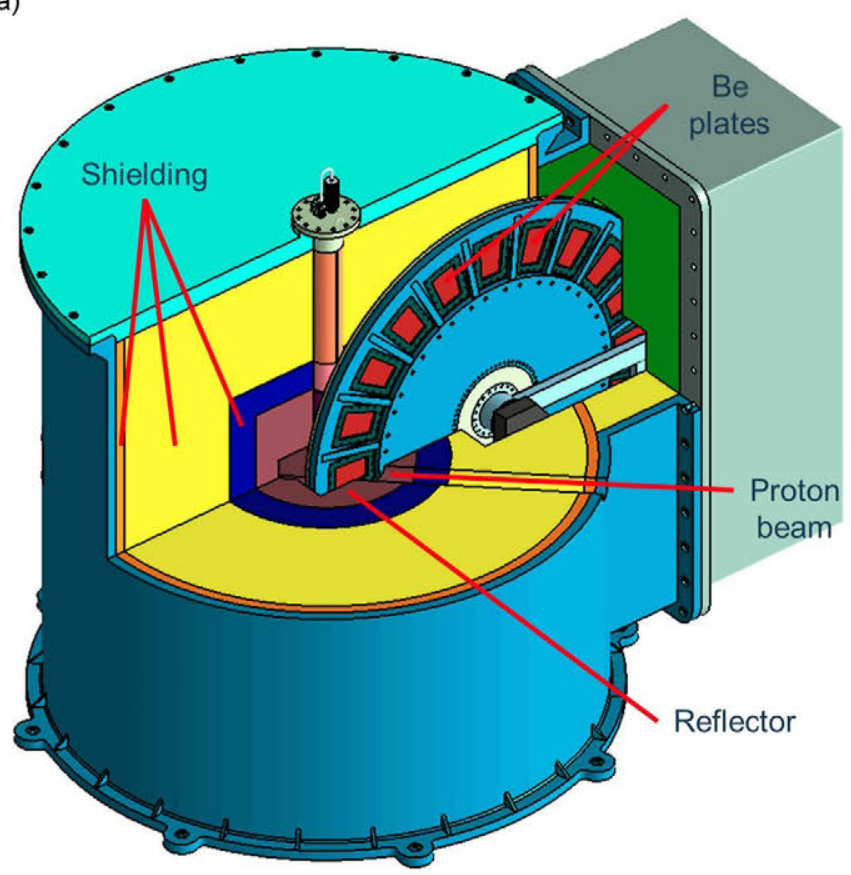

(b)

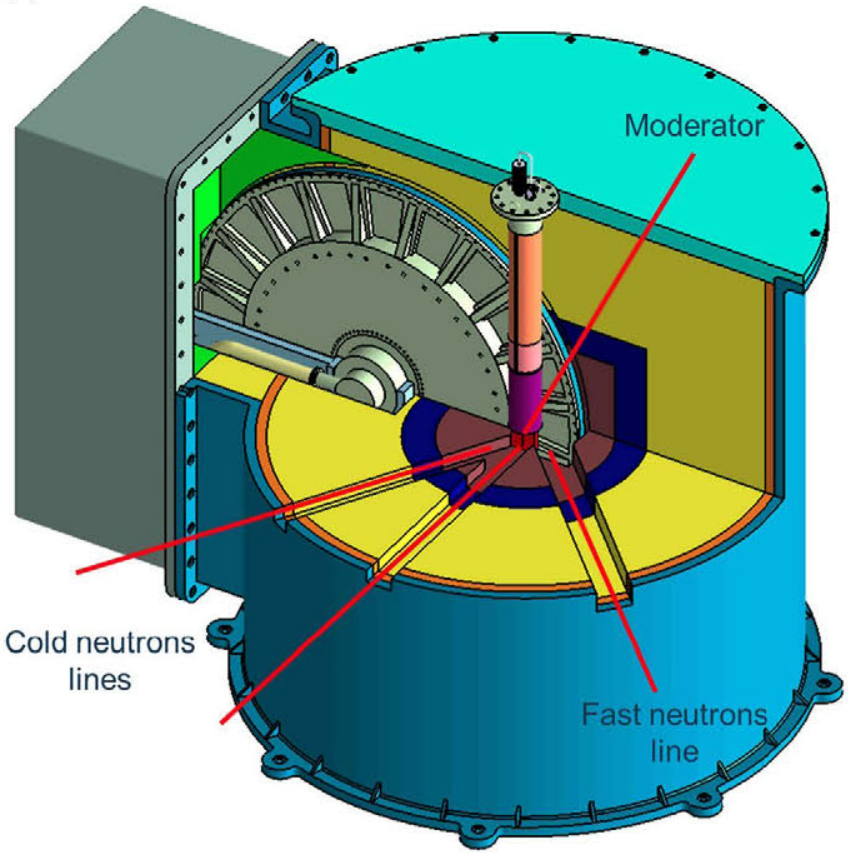

Fig. 3. ESS-Bilbao target station concept.

account the incidence angle, the peak will appear at depths of some $11 \mathrm{~mm}$ within the plate. Most of the neutron production processes take place within the first millimetres of beryllium. A large fraction of the proton energy is however released in the last part of its trajectory. These two facts make it advisable to keep the plate thickness with values smaller than those where the Bragg peak appears, since this will alleviate the heat load without a concomitant reduction of neutron brightness. To illustrate such effects, Table 3 shows the dependence of the heat load during the pulse, temperature, stress and neutron yield on the plate thickness. These results are obtained modeling the reference proton beam in terms of a Gaussian particle distribution with $\sigma=2.5 \mathrm{~cm}$ and $10 \mathrm{~cm}$ in diameter, which is a somewhat conservative option 
Table 3

Effect of Be target thickness on the heat load during pulse, temperature, stress and neutron yield.

\begin{tabular}{clccl}
\hline $\begin{array}{l}\text { Thickness } \\
(\mathrm{mm})\end{array}$ & $\begin{array}{l}\text { Pulse energy dep. } \\
(\mathrm{MW})\end{array}$ & $\begin{array}{l}\mathrm{T} \text { max } \\
\left({ }^{\circ} \mathrm{C}\right)\end{array}$ & $\begin{array}{l}\text { Stress max } \\
(\mathrm{MPa})\end{array}$ & $\begin{array}{l}\text { Neutron yield. } \\
(\mathrm{n} / \mathrm{p})\end{array}$ \\
\hline 6 & 1.43 & 80.0 & 72.2 & $3.9 \times 10^{-2}$ \\
7 & 1.73 & 87.7 & 82.3 & $4.5 \times 10^{-2}$ \\
8 & 2.06 & 95.2 & 93.0 & $5.2 \times 10^{-2}$ \\
9 & 2.44 & 106.3 & 105.4 & $5.7 \times 10^{-2}$ \\
10 & 2.93 & 128.0 & 144.1 & $6.3 \times 10^{-2}$ \\
11 & 3.50 & 142.15 & 168.7 & $6.5 \times 10^{-2}$ \\
12 & 3.58 & 147.3 & 189.9 & $6.5 \times 10^{-2}$ \\
\hline
\end{tabular}

since a more flat profile could be expected. As regards the cooling conditions, a heat-transfer coefficient $h=10^{4} \mathrm{~W} / \mathrm{m}^{2} \mathrm{~K}$ and a coolant temperature of $25^{\circ} \mathrm{C}$ are considered. For the mechanical analysis, the plates have no mechanical restriction, an assumption made in order to make the analysis independent of the mechanical attachment system. The procedure dealing with the fastening of the plates will be defined later during detail engineering activities and the optimal plate geometry will then be analyzed under realistic boundary conditions. Be S-200F grade properties [17] have been considered for thermal and mechanical evaluation purposes in this work. The temperature and stress results listed in the table represent the maximum values reached during the pulse transient representing normal operational conditions for the reference beam and disk configuration (i.e. beam frequency $=20 \mathrm{~Hz}$ and number of beryllium plates $=20$ ).

A glance at Table 3 thus shows that the neutron yield remains constant for plate thicknesses comparable or larger than that where the Bragg peak appears, whereas the maximum temperature and stress sharply increase for plates thicker than $9 \mathrm{~mm}$. This is due to the rise of volumetric heat load within the beryllium plate as the thickness increases up to a value where the Bragg peak will appear. According to this, the thickness of the target plates has been set to $9 \mathrm{~mm}$. Fig. 4 shows the heat load distribution present in a beryllium plate which is thick enough to completely stop the protons. The selection of the $9 \mathrm{~mm}$ thickness avoids the Bragg peak heat load, where the volumetric power would reach up to $152 \mathrm{~kW} / \mathrm{cm}^{3}$ during the pulse.

The number of $9 \mathrm{~mm}$ plates to be held in the disk, i.e. the effect of the beam impact frequency over each plate, is analyzed next under the same thermo-mechanical boundary conditions than those used for the optimization of the plate thickness. The analysis obviously shows that the higher the impact frequency is, the higher the temperatures and stress become. (Figs. 5 and 6). Impact frequencies faster than $1 \mathrm{~Hz}$ involve reaching temperatures that would require significant water pressurization in order to avoid boiling, so the chosen number of beryllium plates for the ESSBilbao target is set to 20 . For this number of plates, the maximum temperature within beryllium remains lower than $107^{\circ} \mathrm{C}$. This temperature is obviously far below than the beryllium melting point $\left(1285^{\circ} \mathrm{C}\right)$ and also far from inducing significant changes on its mechanical properties. Since the maximum water temperature will be lower than this value, a water pressure of 5 bar is chosen to operate while keeping a conservative margin up to boiling ( $\mathrm{T}_{\text {boiling }}$ at 5 bar $=152{ }^{\circ} \mathrm{C}$ ). The maximum stress associated to the thermal gradients is $\approx 105 \mathrm{MPa}$, which is lower than the yield limits of the preselected beryllium commercial grades, S-65 and S-200F at the operation temperatures (207 MPa and $241 \mathrm{MPa}$ respectively) [18]. This stress level is also lower than the beryllium fatigue strength (262 MPa at $10^{7}$ cycles for S-200F) [17], even if the maximum stress value is considered as the cyclic stress.

As far as the damage due to the generation of gas within the material is concerned, the two main sources of it are the

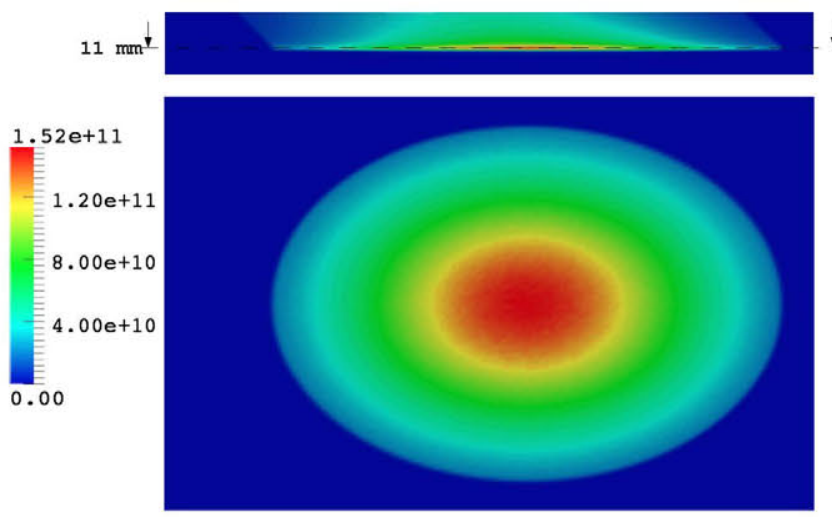

Fig. 4. Heat deposition during pulse due to a ESS-Bilbao proton beam completely stopped in $\mathrm{Be}\left(\mathrm{W} / \mathrm{m}^{3}\right)$

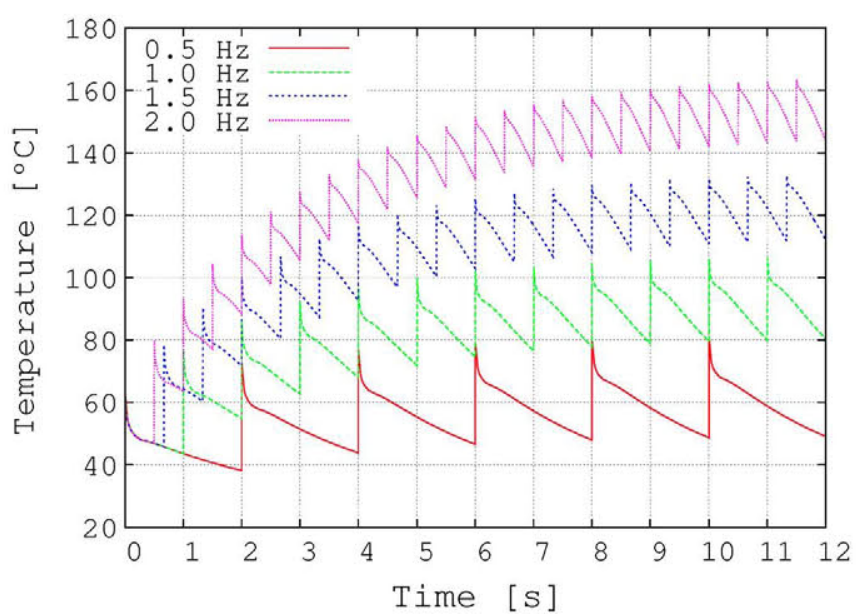

Fig. 5. Maximum temperature evolution for different plates number.

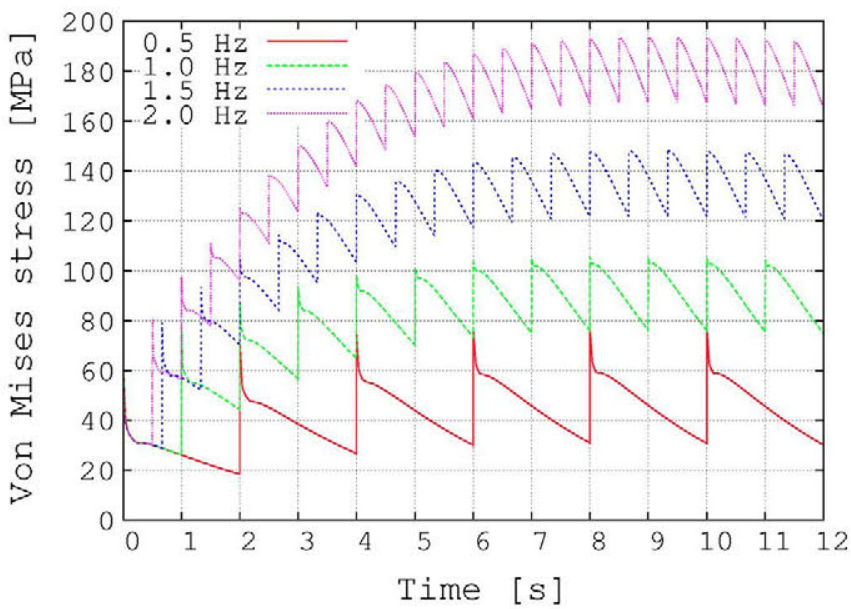

Fig. 6. Maximum von Mises stress evolution for different plates number.

implanted protons and the hydrogen and helium generated by nuclear reactions. Protons from the impinging beam coming to rest within the beryllium plate can later recombine capturing an electron and thus forming atomic hydrogen. Fig. 7 shows the final location of stopped protons resulting from MCNPX simulations (energy cut-off on $1 \mathrm{MeV}$ ) which is representative of the implantation profile on the target. Since most of the protons stop in a very narrow layer close to the Bragg peak, the amount of gas accumulated could destroy the plate after a few weeks of operation. This effect has been already reported from other installations such as LENS where a reduction of the target thickness was adopted as 


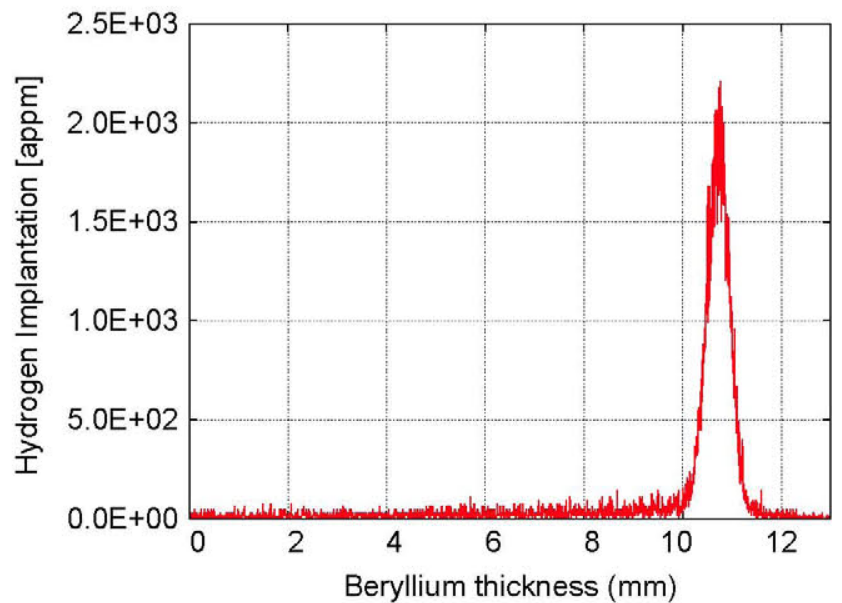

Fig. 7. Hydrogen implantation for $2000 \mathrm{~h}$ of operation.

Table 4

Maximum values of gas generation in $9 \mathrm{~mm}$ beryllium plates estimated with MCNPX $v 2.7$ e code [24] for 2000 operation hours.

\begin{tabular}{lccrrr}
\hline Model & ${ }^{1} \mathrm{H}$ & ${ }^{2} \mathrm{H}$ & ${ }^{3} \mathrm{H}$ & ${ }^{3} \mathrm{He}$ & ${ }^{4} \mathrm{He}$ \\
\hline Bertini-ABLA & 147 & 25 & 5 & 3 & 139 \\
Bertini-Dresner & 147 & 25 & 5 & 3 & 139 \\
CEM03 & 128 & 15 & 12 & 13 & 84 \\
INCL4-ABLA & 130 & 27 & 16 & 13 & 119 \\
INCL4-Dresner & 132 & 28 & 16 & 13 & 120 \\
ISABEL-ABLA & 154 & 18 & 3 & 2 & 139 \\
ISABEL-Dresner & 155 & 18 & 3 & 2 & 140 \\
\hline
\end{tabular}

solution [19]. For a $9 \mathrm{~mm}$ thick plate, the hydrogen implantation comes to be about 170 appm after one year of operation (i.e. 2000 full power hours, considered as representative for a development and training facility). Because of the accelerator characteristics, off-normal low proton energy operation with sizable proton currents, that could completely implant the proton beam in the $9 \mathrm{~mm}$ plate, during long enough to affect the plate integrity is very unlikely. Reaching relevant hydrogen concentrations will require days of undetected malfunction. Since no immediate detection is necessary, normal monitoring of the accelerator parameters, neutron flux, etc., would reveal this off-normal operation soon enough to avoid plate damage.

Table 4 shows the predicted helium and hydrogen generation due to nuclear reactions calculated by means of different nuclear models, considering the average current that each plate will take over a year of operation. The values of generated hydrogen ( $\approx 155 \mathrm{appm})$ are in the same range than the implanted hydrogen. According to [20], this level of hydrogen concentration is no matter of concern and it will partially diffuse out of the beryllium. Nevertheless, helium will stay in the material leading, in the long run to swelling and deterioration of the mechanical properties of the material. The maximum expected accumulation of ${ }^{4} \mathrm{He}$ is below $150 \mathrm{appm} / \mathrm{year}$, so the material will not lose mechanical properties to a significant degree before $3 \mathrm{yr}$ of full power operation $[21,22]$ and the swelling effect will be negligible up to $4 \mathrm{yr}$ [23]. This level of gas implantation/generation damage allows the $9 \mathrm{~mm}$ plates to have an acceptable lifespan.

\subsection{Target disk}

The target disk has been designed to perform two main tasks that are to provide the coolant to the beryllium elements, and to withstand the mechanical loads.
Each beryllium plate will have a $5 \mathrm{~mm}$ thick water channel under it. These channels will be machined within the structural components, and the water volume will be defined after the assembling of all of them. The central distributing/collecting hub will be made of SS316 in order to have a good mechanical support for the target. The material selected for the other components is the 6061-T6 Aluminum alloy, due to its adequate mechanical properties, good workability, high resistance to corrosion and its reduced activation and neutron absorption. SS316 and 6061-T6 thermal and mechanical properties used in this work are referenced in Refs. [25] and [26].

To ensure a heat transfer coefficient of $10^{4} \mathrm{~W} / \mathrm{m}^{2} \mathrm{~K}$ considered during the beryllium plates design, a flow velocity of about $2.25 \mathrm{~m} / \mathrm{s}$ through the channels is required on the grounds of the Dittus-Boelter and Sieder-Tate correlations (the channel crosssection is $\approx 5 \times 10^{-3} \mathrm{~m} \times 10^{-1} \mathrm{~m}$ ). The CFD model developed implements an RNG $\kappa-\epsilon$ turbulent model with wall functions and takes into account buoyancy and the rotatory dynamic effects. The water properties used are referenced in Ref. [27]. The design of the channels guarantees a homogeneous flow velocity of $\approx 2.5 \mathrm{~m} / \mathrm{s}$ (Fig. 8), being the total volumetric flow of the target $26 \mathrm{l} / \mathrm{s}$. The pressure drop in the disk is around 0.6 bar. The transient simulation shows a maximum temperature for the water of around $105^{\circ} \mathrm{C}$ (Fig. 9), coherent with the thermal analysis of the plates. No short-term erosion is expected given the flow velocities involved.

In order to evaluate the disk capacity to withstand the main mechanical loads, inner pressure and thermo-mechanical stress, a steady-state mechanical model of the assembly has been developed. The bolts distribution is designed to meet the mechanical requirements and the bolts grade and preload defined according to [28]. The bolts have been modeled by means of beam elements, and constraint relationships between them and nodes of the structural components have been defined in order to transmit the bolting preload. The materials behavior is considered purely elastic. The interaction between the components has been modeled by means of a frictional augmented Lagrange contact formulation.

To take into account the thermal effects, $5.6 \mathrm{~kW}$ (i.e. $112 \mathrm{~kW} /$ 20 plates) of heating has been considered in the beryllium plate. Given its volume, a uniform $18.86 \mathrm{MW} / \mathrm{m}^{3}$ volumetric power source has been implemented. This simplification provides us a representative temperature distribution of the disk structure, used to obtain a first approach to the ensuing thermo-mechanical effects on the assembly. Considering perfect thermal contact between all the components and a coolant inlet temperature of $25^{\circ} \mathrm{C}$, the maximum of the temperature distribution is $\approx 55^{\circ} \mathrm{C}$ in the beryllium plate (Fig. 10).

The temperature distribution shown in Fig. 10 is then used in the mechanical model, together with the inner pressure of 5 bar, as well as the effect of the bolts preloading. The mechanical inertial effects are negligible due to the small angular velocities and accelerations involved.

The deformation under these loads is found to reach acceptable values, with the maximum displacement below $0.5 \mathrm{~mm}$ (Fig. 11). This deformation is admissible since the channel water velocity would remain at $\approx 90 \%$ of its nominal value and it could be easily further reduced, if necessary, by the plate attachment system. Regarding the stress, and disregarding the non-representative values in the sharp edges, the maximum stress in the aluminium alloy remains below $70 \mathrm{MPa}$ (Fig. 12). This value is lower than the 6061-T6 alloy yield stress (250 MPa) and also lower than the fatigue limit (100 MPa, for $5 \times 10^{8}$ completely reversed cycles [26]). The maximum stress in the beryllium for this steady state analysis is $\approx 50 \mathrm{MPa}$. This value is mainly due to the different thermal expansion coefficients of the aluminium alloy $\left(23.5 \times 10^{-6}\right.$ $\left.\mathrm{K}^{-1}\right)$ and beryllium $\left(11.5 \times 10^{-6} \mathrm{~K}^{-1}\right)$ and to the perfect fitting 


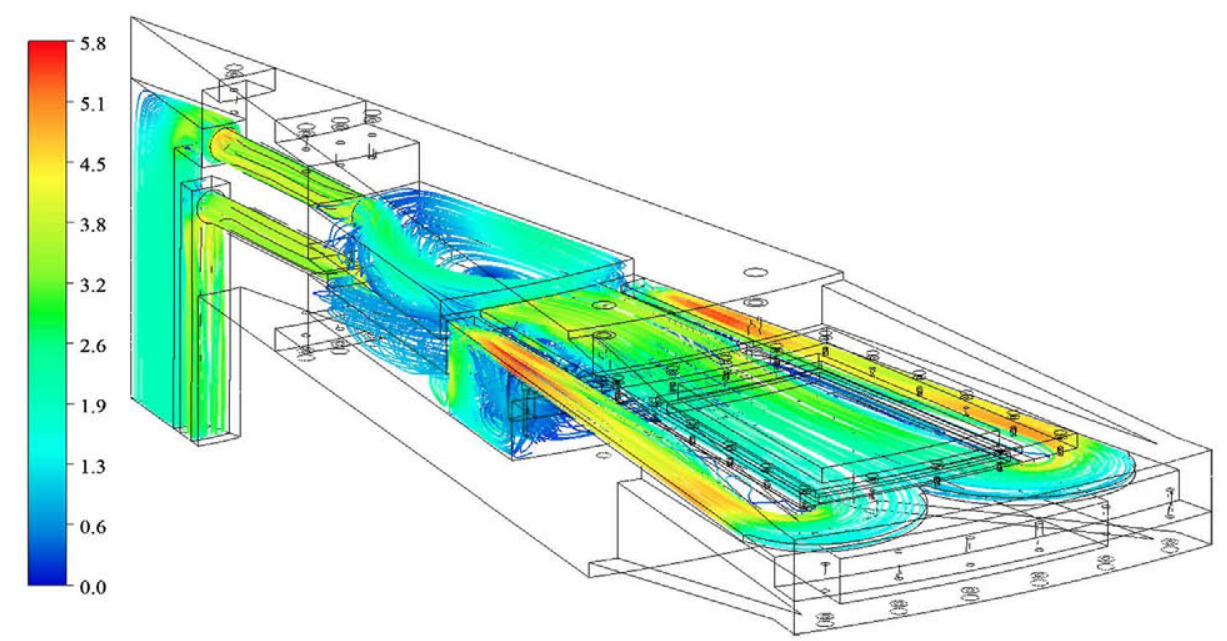

Fig. 8. Water flow velocities $(\mathrm{m} / \mathrm{s})$ in a fluid sector.

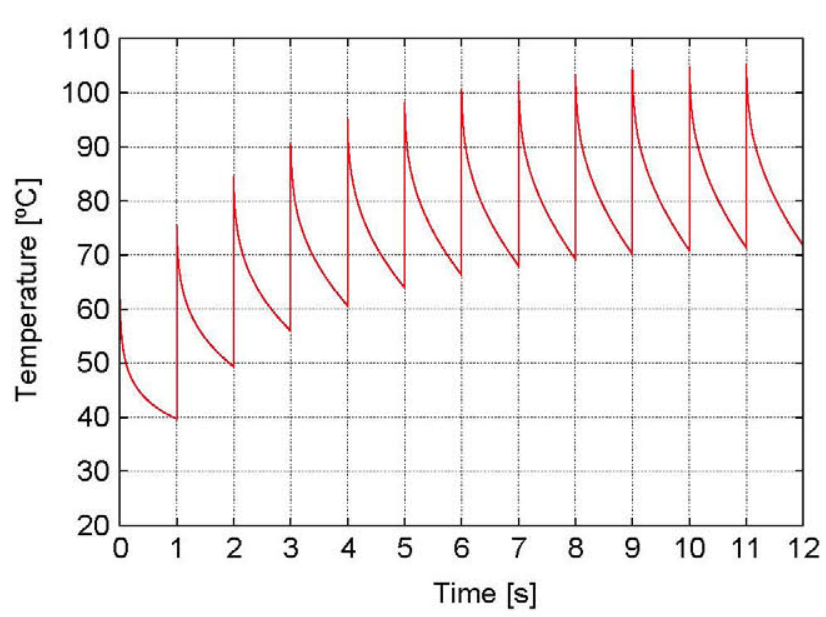

Fig. 9. Maximum water temperature.

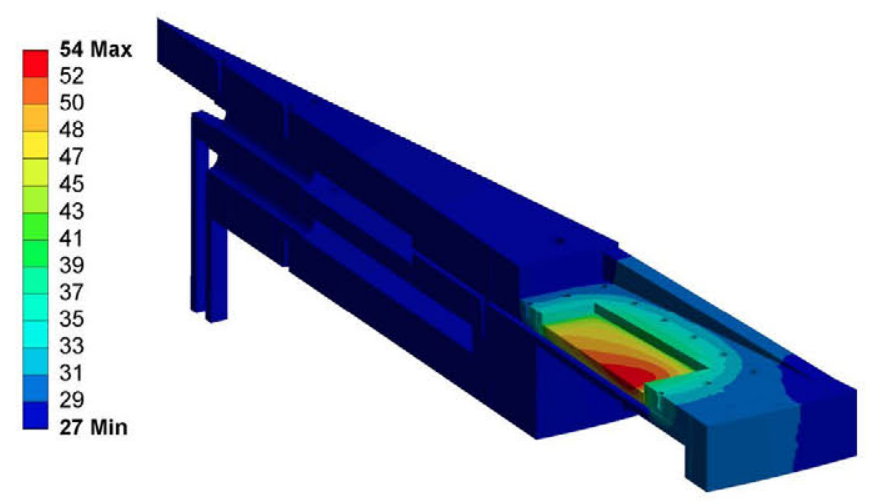

Fig. 10. Temperatures $\left({ }^{\circ} \mathrm{C}\right)$ in a disk sector for an averaged power source.

between the plates and the disk assumed in this model. The foreseen joint between these components will be a flanged seal, able to accommodate some differential dilatation.

Corrosion on aluminum and beryllium, in the normal operation temperature range, can be avoid if the $\mathrm{pH}$ of water is in between 4 and 8, so chemical control of the cooling circuit will be needed. Possible galvanic corrosion between metals will be controlled by surface treatment and/or cathodic protection [17,29].

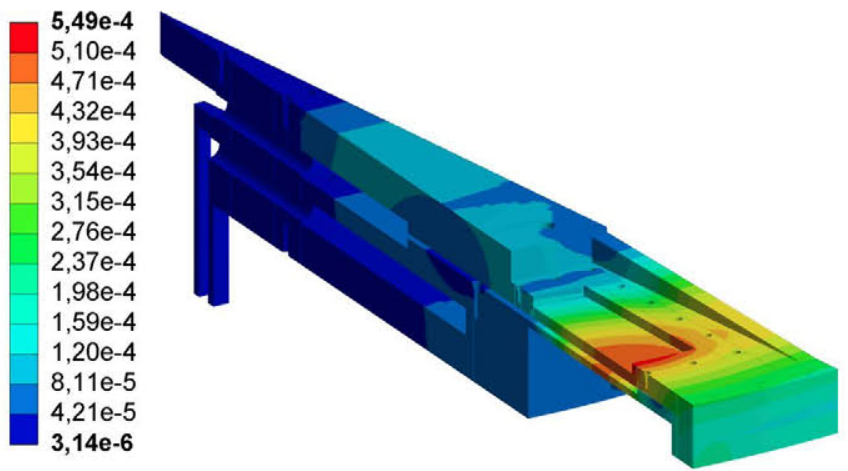

Fig. 11. Total displacements $(\mathrm{m})$ in a disk sector.

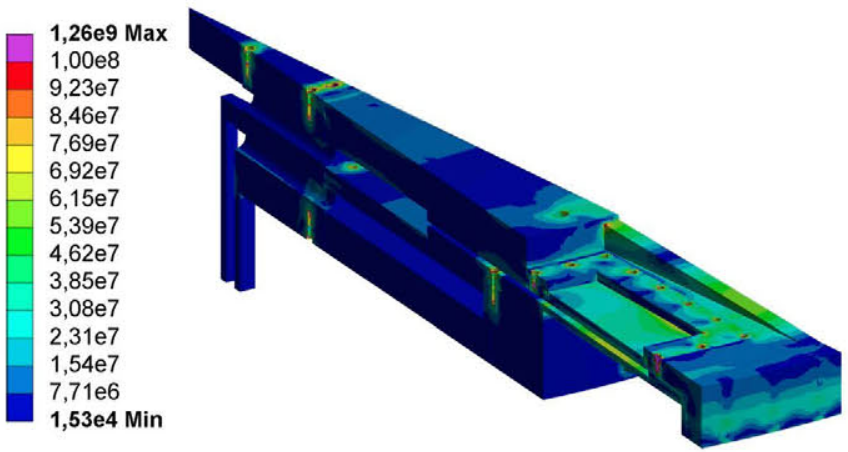

Fig. 12. Von Mises stress $(\mathrm{Pa})$ in a disk sector

\section{Conclusions}

The concept of the ESS-Bilbao neutron source consists of a rotating beryllium target cooled by water. The main structural materials of the target disk are be 6061-T6 aluminium alloy and SS316. The disk holds 20 beryllium plates with a $9 \mathrm{~mm}$ thickness for a beam repetition rate of $20 \mathrm{~Hz}$.

The plates are cooled by water at $2.5 \mathrm{~m} / \mathrm{s}$, flowing through $5 \mathrm{~mm}$ thick cooling channels at a 5 bar pressure. The designed cooling system ensures acceptable levels of temperature and thermal stress in the beryllium plates.

The mechanical loads over the disk structures are withstood without expected fatigue effects. 
The expected lifespan of the beryllium plates, based on the degradation of mechanical properties due to the gas implantation/ generation, is at least three full power years (considering $2000 \mathrm{~h}$ per year).

\section{Acknowledgments}

This work has been possible thanks to the support of the computing infrastructure of the i2BASQUE academic network.

Fruitful discussions with P. Sokol (LENS), as well as with several members of the PSI and ISIS Target Groups are gratefully acknowledged.

\section{References}

[1] David V. Baxter, J. Leung, Helmut Kaiser, S. Ansell, G. Muhrer, E.B. Iverson, P. D. Fergusson, Physics Procedia 26 (2012) 117

[2] David V Baxter, S. Ansell, P.D. Ferguson, F.X. Gallmeier, E.B. Iverson, H. Kaiser, W. Lu, G. Muhrer, T.C. Rinckel, T. Steinbach, Physics Procedia 26 (2012) 153.

[3] M. Ooi, et al., Nuclear Instruments and Methods in Physics Research Section A: Accelerators, Spectrometers, Detectors and Associated Equipment 659 (1) (2011) 61.

[4] IAEA, Development Opportunities for Small and Medium Scale Accelerator Driven Neutron Sources, Report of a Technical Meeting held in Vienna, 18-21 May 2004, 1439 (ISBN:92-0-101705-7), IAEA, 2005.

[5] Report from the ILL Associates Working Group on Neutrons in Europe for 2025, Technical Report, ILL, 2011, 〈www.ill.eu/fileadmin/users_files/docu ments/news and events/news/Report from ILL Neutron Working Group. pdf) (Last accessed April 2013.)

[6] M. Lindroos, et al., Nuclear Instruments and Methods in Physics Research Section B: Beam Interactions with Materials and Atoms 269 (24) (2011) 3258.

[7] F. Sordo, S. Terrón, M. Magán, G. Muhrer, A. Ghiglino, F. Martínez, PJ de Vicente, R. Vivanco, J.M. Perlado, F.J. Bermejo, Nuclear Instruments and Methods in Physics Research Section A: Accelerators, Spectrometers, Detectors and Associated Equipment 707 (2013) 1.

[8] M. Lewitowicz, Nuclear Physics A 734 (0) (2004) 645, Proceedings of the Eighth International Conference on Nucleus-Nucleus Collisions.

[9] A. Andrighetto, L. Biasetto, M. Manzolaro, M. Barbui, G. Bisoffi, S. Carturan, M. Cinausero, F. Gramegna, G. Prete, V. Rizzi, C. Antonucci, S. Cevolani, C. Petrovich, P. Colombo, G. Meneghetti, P. Di Bernardo, P. Zanonato, I. Cristofolini, V. Fontanari, B. Monelli, R. Oboe, Nuclear Instruments and Methods in Physics Research Section B: Beam Interactions with Materials and Atoms 266 (19-20) (2008) 4257.

[10] M.B. Chadwick, P. Obloinska, M. Herman, N.M. Greene, R.D. McKnight, D. L. Smith, P.G. Young, R.E. MacFarlane, G.M. Hale, S.C. Frankle, A.C. Kahler, T. Kawano, R.C. Little, D.G. Madland, P. Moller, R.D. Mosteller, P.R. Page, P. Talou, H. Trellue, M.C. White, W.B. Wilson, R. Arcilla, C.L. Dunford, S. F. Mughabghab, B. Pritychenko, D. Rochman, A.A. Sonzogni, C.R. Lubitz, T.
H. Trumbull, J.P. Weinman, D.A. Brown, D.E. Cullen, D.P. Heinrichs, D. P. McNabb, H. Derrien, M.E. Dunn, N.M. Larson, L.C. Leal, A.D. Carlson, R. C. Block, J.B. Briggs, E.T. Cheng, H.C. Huria, M.L. Zerkle, K.S. Kozier, A. Courcelle, V. Pronyaev, S.C. van der Marck, Nuclear Data Sheets 107 (12) (2006) 2931, Evaluated Nuclear Data File ENDF/B-VII.O.

[11] K. Thomsen, L. Buligins, M. Butzek, G. Heidenreich, F. Heinrich, M.Magán, E. Pitcher. Target cooling options for neutron sources at high power densities. Proceedings of third Union for Compact Accelerator-driven Neutron Sources meeting, 2012.

[12] M.B. Leuschner, D.V. Baxter, V.P. Derunchuk, H. Kaiser, C.M. Lavelle, H. Nann, N.B. Remmes, T. Rinckel, W.M. Snow, P.E. Sokol, Nuclear Instruments and Methods in Physics Research Section B: Beam Interactions with Materials and Atoms 261 (1-2) (2007) 956

[13] Masashi Takada, Erika Mihara, Takashi Nakamura, Toshihiko Honma, Koji Kono, Kazunobu Fujitaka, Nuclear Instruments and Methods in Physics Research Section A: Accelerators, Spectrometers, Detectors and Associated Equipment 545 (3) (2005) 765.

[14] T. McManamy, M. Rennich, F. Gallmeier, P. Ferguson, J. Janney, Journal of Nuclear Materials 398 (1-3) (2010) 35, Proceedings of the Ninth International Workshop on Spallation Materials Technology.

[15] G.S. Bauer, Design Studies for a Rotating Target Arrangement for a Spallation Neutron Source, Technical Report, Report Jül-Conf-34, KFA Jülich, 1980, pp. 175.

16] A. Ghiglino, M. Magán, A. Zarraoa-Garmendia, B. Riemer, F. Sordo, A. Abdoud, T.J. McManamy, F.J. Bermejo, J.M. Perlado, Tests on the SNS Rotating Target design at the RTFT ESS BILBAO, Ghent, Belgium, 2012.

[17] Beryllium Chemistry and Procesing, ASM International, 2009, ISBN-13: 9780871707215.

[18] Materion brush beryllium \& composites, Designing and Fabricating beryllium, Technical Report, 〈http://materion.com/ResourceCenter/ProductData/Beryl lium/BerylliumMetal/DesignGuides.aspx >.

[19] T.C. Rinckel, P.E. Sokol, Internal Report LENS (2006).

[20] D. Filges, F. Goldenbaum, Handbook of Spallation Research: Theory, Experiments and Applications, Wiley-VCH, 2010.

[21] L. Sannen, C. De Raedt, F. Moons, Y. Yao, Fusion Engineering and Design 29 (1995) 470.

[22] F. Moons, L. Sannen, A. Rahn, J. Van De Velde, Journal of Nuclear Materials 233 (1996) 823.

[23] F. Scaffidi-Argentina, G.R. Longhurst, V. Shestakov, H. Kawamura, Fusion Engineering and Design $51(2000) 23$

[24] MCNPX user's Manual, Version 2.6.0. 2007.

[25] ASM Handbook Volume 01: Properties and Selection: Irons, Steels, and HighPerformance Alloys. ASM International, 1990, ISBN-13: 9780871703774.

26] ASM Handbook Volume 02: Properties and Selection: Nonferrous Alloys and Special-Purpose Materials, ASM International, 1990, ISBN-13: 9780871703781

[27] Revised Release on the IAPWS Industrial Formulation 1997 for the Thermodynamic Properties of Water and Steam (The revision only relates to the extension of region 5 to $50 \mathrm{MPa}$.), IAPWS, 2007.

[28] Richard G. Budynas, J. Keith Nisbett, Shigley's Mechanical Engineering Design, 8th ed., McGraw-Hill, 2006, ISBN-13: 9780073312606.

[29] G.E. Totten, D.S. MacKenzie (Eds.), Handbook of Aluminum, Alloy Production and Materials Manufacturing, CRC Press, vol. 2, 2003, ISBN-13: 9780824708962. 